\title{
Ferroelectric Transition in Vinylidene-Trifluoroethylene Copolymer Studied by Nuclear Magnetic Resonance Method
}

\author{
Fumiaki ISHII, Akira OdAJima, and Hiroji OHIGASHI* \\ Department of Applied Physics, Faculty of Engineering, \\ Hokkaido University, Sapporo 060, Japan \\ *Engineering Research Laboratories, Toray Industries Inc., \\ Tebiro, 1111, Kamakura, Kanagawa
}

(Received June 29, 1983)

\begin{abstract}
NMR absorption lines for a uniaxially drawn film of VDF-TrFE copolymer (72:28) were measured as a function of the alignment angle $\gamma$ between the drawn axis and the magnetic field, over a temperature range from 23 to $130^{\circ} \mathrm{C}$. The line shape consisted of inner, intermediate and outer peaks below the Curie temperature $T_{\mathrm{c}}$. The line width of the intermediate peak narrowed at about $40^{\circ} \mathrm{C}$ with increasing temperature, and above $80^{\circ} \mathrm{C}$ it increased gradually by $1-3$ Oe up to $T_{\mathrm{c}}$. This line width as well as the line width of the outer peak strongly depended on $\gamma$, indicating that the nuclear dipole interactions in the crystalline region contribute to these peaks. Above $T_{c}$ the width of the outer peak and the second moment decreased markedly owing to the chain motion accompanying conformational changes from the all-trans sequence to the combination of TG, TG, $T_{3} G$, and $T_{3} \bar{G}$ groups. The remarkable $\gamma$-dependence of the line shape as well as the line width observed in the paraelectric phase may be interpreted by a one-dimensional diffusion motion of conformational defects, and compared well with the ESR of a one-dimensional magnetic substance $\left(\mathrm{CH}_{3}\right)_{4} \mathrm{NMnCl}_{3}$ (TMMC).

KEY WORDS Ferroelectric Transition / VDF-TrFE Copolymer / NMR /

Conformational Defects / $\gamma$-Dependence of Second Moment /
\end{abstract}

Ferroelectric characteristics of vinylidenetrifluoroethylene copolymer $(\mathrm{P}(\mathrm{VDF} / \mathrm{TrFE}))$ is closely related with its crystal structure and chain conformation. Davis et al. ${ }^{1}$ reported that above the Curie temperature $\left(T_{\mathrm{c}}\right)$ the all-trans conformation of chains in the orthorhombic (psuedo-hexagonal) lattice changes to the disordered 3/1-helical one in the hexagonal latticelike polytrifluoroethylene (PTrFE). On the other hand, Tashiro et al.'s IR and $X$-ray measurements ${ }^{2}$ showed that the chain deflected with an angle of $\pm 15^{\circ}$ in the orthorhombic unit cell changes to a chain with random sequences of $\mathrm{TG}, \mathrm{TG}, \mathrm{T}_{3} \mathrm{G}$ and $\mathrm{T}_{3} \overline{\mathrm{G}}$ groups in the paraelectric phase. They suggested that this conformational change is closely related to the dielectric anomalies near $T_{\mathrm{c}}$ reported by Furukawa et $a l^{3}$

This paper reports the molecular chain motions in a drawn $\mathrm{P}(\mathrm{VDF} / \mathrm{TrFE})$ film of high crystallinity studied by the wide line-nuclear resonance (NMR) technique. The transition mechanism in the ferro- and paraelectric phases was also investigated on the basis of the motional characteristics of the chain in each phase.

\section{EXPERIMENTAL}

The samples used were films of a random copolymer VDF-TrFE $(72: 28 \mathrm{~mol} \%)$, supplied by Daikin Kogyo Co., Ltd. The film was drawn $\times 4$ uniaxially at room temperature and annealed at $135^{\circ} \mathrm{C}$ for $9 \mathrm{~h}$. Its final drawn ratio was 3.5 and its thickness was $100 \mu$.

NMR absorption was measured for proton-spins in $\mathrm{P}(\mathrm{VDF} / \mathrm{TrFE})$ using a JNM-WS-40 NMR spectrometer operating at a resonance frequency of $40 \mathrm{MHz}$. The modulation field was $35 \mathrm{~Hz}$ and the amplitude from 1 to $0.2 \mathrm{Oe}$. A weak level of radiofrequency power was selected in order to avoid the saturation effect. Figure 1 illustrates the TNM coordinates fixed to the sample. 


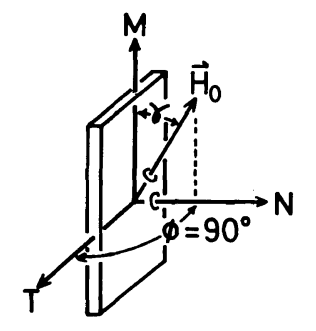

Figure 1. TNM coordinates fixed in the drawn film. The direction of the magnetic field $\vec{H}_{0}$ is specified by the alignment angle $\gamma$.

The magnetic field $\vec{H}_{0}$ applied was in the plane containing the drawn axis and the normal axis $(N$ axis), and the alignment angle $\gamma$ between $\vec{H}_{0}$ and the $M$-axis was varied from 0 to $90^{\circ}$ at constant temperatures ranging from 23 to $130^{\circ} \mathrm{C}$.

\section{RESULTS}

\section{NMR Line Shape}

Figure 2 shows the right side of the NMR derivative curves for $\mathrm{P}(\mathrm{VDF} / \mathrm{TrFE})$ measured at various temperatures for $\gamma=0,45$ (or 55), and $90^{\circ}$. The arrows $\uparrow$ and $\downarrow$ indicate the temperature rise from 23 to $130^{\circ} \mathrm{C}$ and fall from 130 to $23^{\circ} \mathrm{C}$, respectively. Three peaks are observed in the curves for $\gamma=0^{\circ}$ and $90^{\circ}$ at $T=23$ to $107^{\circ} \mathrm{C} \uparrow$, which correspond to the broad, intermediate and narrow widths $\left(\Delta H_{\mathrm{b}}, \Delta H_{\mathrm{m}}\right.$, and $\left.\Delta H_{\mathrm{s}}\right)$. Above $118^{\circ} \mathrm{C}$ in the $\uparrow-$ process or above $78^{\circ} \mathrm{C}$ in the $\downarrow$-process, the derivative curves changed suddenly to two peaks having broad and narrow widths $\left(\Delta H_{\mathrm{b}}^{\prime}\right.$ and $\left.\Delta H_{\mathrm{s}}^{\prime}\right)$, as seen for the $127^{\circ} \uparrow$ and $80^{\circ} \mathrm{C} \downarrow$ curves in Figure 2. It is clear from this figure that both broad widths $\left(\Delta H_{\mathrm{b}}\right.$ and $\left.\Delta H_{\mathrm{b}}^{\prime}\right)$ depend on the alignment angle $\gamma$, while neither narrow one $\left(\Delta H_{\mathrm{s}}\right.$ and $\left.\Delta H_{\mathrm{s}}\right)$ does.

\section{Line Width and Its Temperature Dependence}

Figures $3 \mathrm{a}, 3 \mathrm{~b}$, and $3 \mathrm{c}$ show the line widths plotted against temperature for $\gamma=0,45$, and $90^{\circ}$, respectively. The broad width for $\gamma=90^{\circ}$ narrows in the temperature region from 80 to $114^{\circ} \mathrm{C}$ and narrows again abruptly in the region from 114 to $118^{\circ} \mathrm{C}$, while the broad widths for $\gamma=0$ and $45^{\circ}$ narrow only above $114^{\circ} \mathrm{C}$. The line widths hardly change with increasing temperature in the region from 118 to $130^{\circ} \mathrm{C}$, but still depend on the alignment angle $\gamma$. These three temperature regions are a)
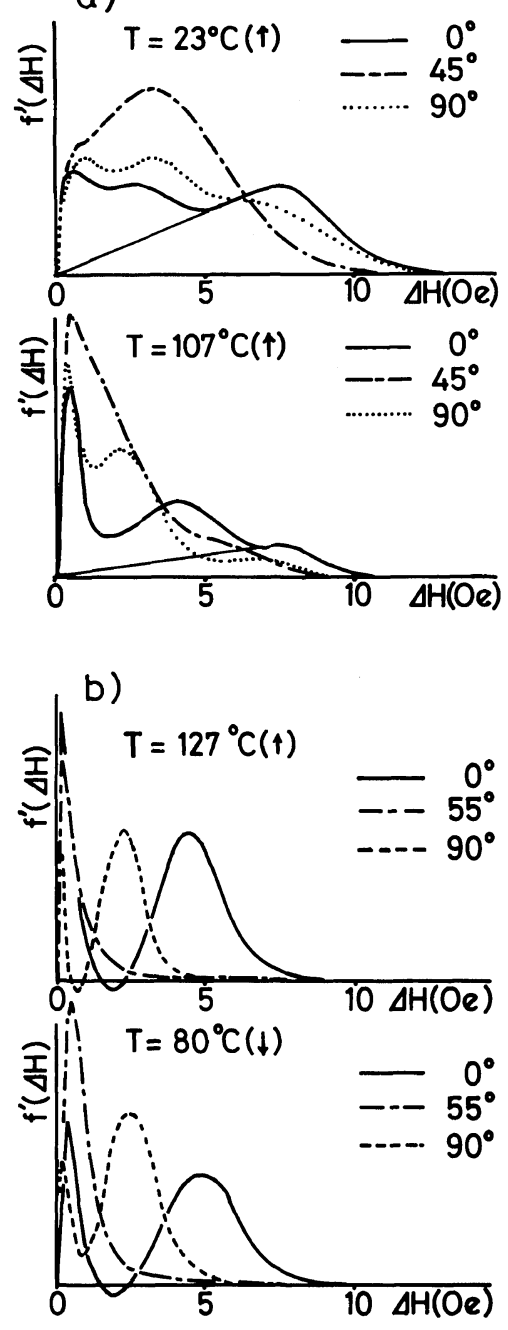

Figure 2. Derivative curves of NMR absorption line of $\mathrm{P}(\mathrm{VDF} / \mathrm{TrFE})-72 / 28$ for 0,45 , and $90^{\circ}$ of the alignment angle $\gamma$ between the magnetic field and the drawn axis; (a) $23^{\circ} \mathrm{C} \uparrow$ and $107^{\circ} \mathrm{C} \uparrow$; (b) $127^{\circ} \mathrm{C} \uparrow$ and $80^{\circ} \mathrm{C} \downarrow$.

referred to as the $\alpha_{b} \uparrow, \alpha_{t} \uparrow$, and $\alpha_{b}^{\prime} \uparrow$ regions, respectively, in the order of increasing temperature.

Since the Curie temperature $T_{\mathrm{c}}$ can not be determined exactly from the narrowing in the $\alpha_{t} \uparrow$ region, it is represented by an averaged temperature, that is, $T_{\mathrm{c}} \uparrow=116^{\circ} \mathrm{C}$ in the $\alpha_{\mathrm{t}} \uparrow$ region. The intermediate widths $\Delta H_{\mathrm{m}}$ 's for $\gamma=0,45$, and $90^{\circ}$ decrease gradually in the temperature region between 30 and $80^{\circ} \mathrm{C}$, and increase gradually from 80 to $114^{\circ} \mathrm{C}$ in the $\alpha_{b} \uparrow$ region. These narrowing and 

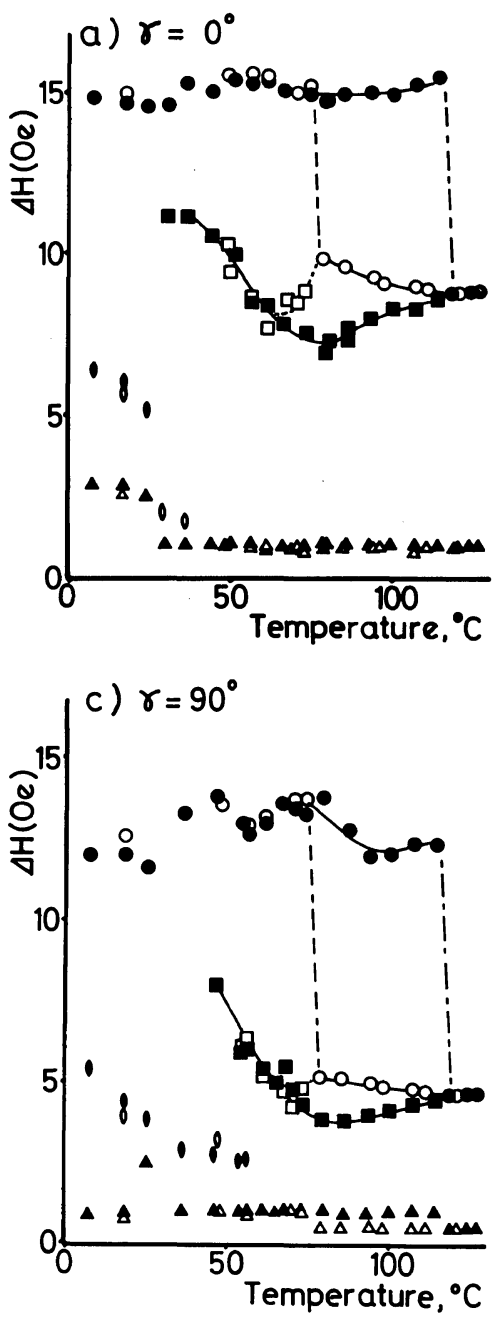

broadening regions of the intermediate width are referred to as the $\alpha_{\mathrm{m}} \uparrow$ and $\beta_{\mathrm{m}} \uparrow$ regions, respectively. It seems that $\Delta H_{\mathrm{m}}(\gamma)$ approaches $\Delta H_{\mathrm{b}}^{\prime}(\gamma)$ above $118^{\circ} \mathrm{C}$, except for $\gamma=45^{\circ}$.

A broadening of $2-3 \mathrm{Oe}$ is observed for $\Delta H_{\mathrm{b}}^{\prime}(\gamma)$ 's between 130 and $78^{\circ} \mathrm{C}$, which is referred to as the $\alpha_{b}^{\prime} \downarrow$ region. Below the $\alpha_{b}^{\prime} \downarrow$ region, the line width broadens abruptly between 78 and $74^{\circ} \mathrm{C}$, which is referred to as the $\alpha_{t}^{\prime} \downarrow$ region, and then returns to the $\Delta H_{\mathrm{b}}(\gamma)$ value in the $\uparrow$-process. The intermediate width ( $\square$ ) simultaneously appears below $T_{\mathrm{c}} \downarrow$, decreases rapidly in the region from 74 to $61^{\circ} \mathrm{C}$, which is referred to as the $\beta_{\mathrm{m}}^{\prime} \downarrow$ region, and then returns to the $\Delta H_{\mathrm{m}}$ value in the $\uparrow$-process.

The $\gamma$-dependences of $\Delta H_{\mathrm{b}}$ and $\Delta H_{\mathrm{m}}$ are shown in Figure 4 at various temperatures. Each curve repre-

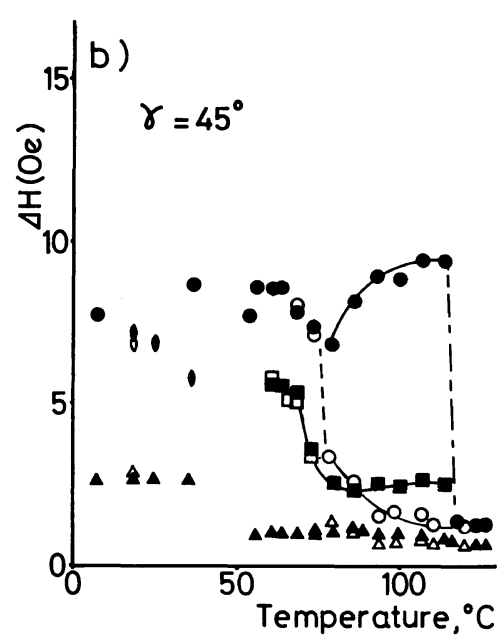

Figure 3. Line widths of $\mathrm{P}(\mathrm{VDF} / \mathrm{TrFE})-72 / 28$ as a function of temperature: (a) $\gamma=0^{\circ}$; (b) $\gamma=45^{\circ}$; (c) $\gamma=90^{\circ}$. The filled circle, square and triangle indicate the broad, intermediate and narrow widths, respectively, in the $\uparrow-$ process. The unfilled circle, square and triangle indicate the broad, intermediate and narrow widths, respectively, in the $\downarrow$-process.

sents a characteristic in the rigid and motional states $\left(\alpha_{\mathrm{m}} \uparrow, \beta_{\mathrm{m}} \uparrow\left(\right.\right.$ or $\left.\alpha_{\mathrm{b}} \uparrow\right), \alpha_{\mathrm{b}}^{\prime} \uparrow$ and $\left.\alpha_{\mathrm{b}}^{\prime} \downarrow\right)$. In the paraelectric phase above $T_{\mathrm{c}} \uparrow$ or $T_{\mathrm{c}} \downarrow$, the angle $\gamma$, at which $\Delta H_{\mathrm{b}}(\gamma)$ shows a minimum, suddenly changes from 45 to $55^{\circ}$, and a valley in the $\Delta H_{\mathrm{b}}(\gamma)$ curve becomes somewhat deeper. In the $\alpha_{b}^{\prime} \downarrow$ region, however, the angle for the minimum $\Delta H_{\mathrm{b}}(\gamma)$ shows little change. In Table I, the temperature ranges for the $\alpha_{\mathrm{m}} \uparrow, \alpha_{\mathrm{b}} \uparrow$ (or $\beta_{\mathrm{m}} \uparrow$ ), $\alpha_{\mathrm{t}} \uparrow, \alpha_{\mathrm{b}}^{\prime} \uparrow, \alpha_{\mathrm{b}}^{\prime} \downarrow, \alpha_{\mathrm{t}}^{\prime} \downarrow$, and $\beta_{\mathrm{m}}^{\prime} \downarrow$ states, and $T_{\mathrm{c}} \uparrow$ and $T_{\mathrm{c}} \downarrow$ are summarized.

\section{NMR Second Moment and Mobile Fraction}

The broad and narrow components in the derivative absorption line were separated by a straight line connecting the origin to the minimum point, as indicated in Figure 2. In this case, the narrow 

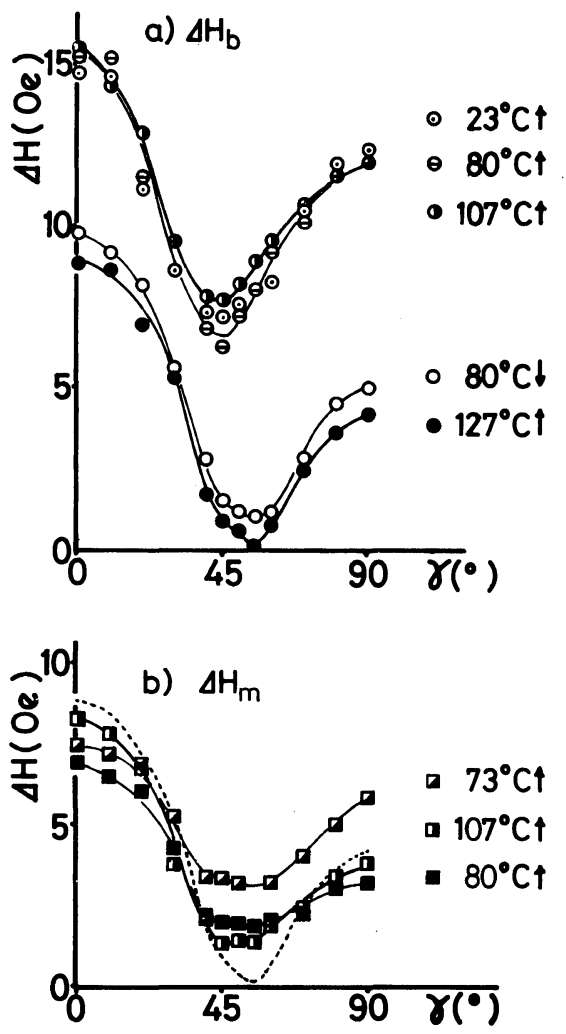

Figure 4. Line withs of $\mathrm{P}(\mathrm{VDF} / \mathrm{TrFE})-72 / 28$ as a function of the alignment angle $\gamma$ at various temperatures: (a) broad width, $\Delta H_{\mathrm{b}}$; (b) intermediate width, $\Delta H_{\mathrm{m}}$.

component consists of intermediate and inner peaks corresponding to the $\Delta H_{\mathrm{m}}$ and $\Delta H_{\mathrm{s}}$, respectively. Figures $5 \mathrm{a}$ and $5 \mathrm{~b}$ show the temperature changes of NMR second moments $\left\langle\Delta H^{2}\right\rangle_{\mathrm{ob}}(\gamma)$ 's. The temperature dependence of the second moments of the broad component, $\left\langle\Delta H^{2}\right\rangle_{\mathrm{b}}\left(0^{\circ}\right)$ and $\left\langle\Delta H^{2}\right\rangle_{\mathrm{b}}\left(90^{\circ}\right)$, is shown in Figure 6. It should be noted that $\left\langle\Delta H^{2}\right\rangle_{\mathrm{ob}}\left(0^{\circ}\right)$ and $\left\langle\Delta H^{2}\right\rangle_{\mathrm{b}}\left(90^{\circ}\right)$ decrease gradually with increasing temperature in the $\alpha_{b} \uparrow$ region, while $\left\langle\Delta H^{2}\right\rangle_{\mathrm{b}}\left(0^{\circ}\right)$ remains unchanged. When the temperature crosses $T_{\mathrm{c}} \uparrow$, the second moment decreases suddenly, but above $T_{\mathrm{c}} \uparrow$, still depends on the alignment angle $\gamma$. It increases slightly (1$\left.3 \mathrm{Oe}^{2}\right)$ in the $\alpha_{\mathrm{b}}^{\prime} \downarrow$ region. Furthermore, when $T_{\mathrm{c}} \downarrow$ is crossed $\left\langle\Delta H^{2}\right\rangle_{\mathrm{ob}}(\gamma)$ suddenly increases and returns to the $\left\langle\Delta H^{2}\right\rangle_{\mathrm{ob}}(\gamma)$ value in the $\uparrow$-process.

In Figure 6 , the fraction $F_{\mathrm{m}}$ of the mobile part in the sample (the mobile fraction) is plotted against
Table I. Classification of motional states and transition temperatures of $\mathrm{P}(\mathrm{VDF} / \mathrm{TrFE})-72 / 28$

\begin{tabular}{|c|c|c|c|}
\hline \multicolumn{2}{|c|}{$\uparrow$-Process } & \multicolumn{2}{|c|}{$\downarrow$-Process } \\
\hline \multirow{2}{*}{$\begin{array}{l}\text { Motional } \\
\text { state }\end{array}$} & $\begin{array}{l}\text { Temperature } \\
\text { region }\end{array}$ & \multirow{2}{*}{$\begin{array}{l}\text { Motional } \\
\text { state }\end{array}$} & $\begin{array}{l}\text { Temperature } \\
\text { region }\end{array}$ \\
\hline & ${ }^{\circ} \mathrm{C}$ & & ${ }^{\circ} \mathrm{C}$ \\
\hline$\alpha_{\mathrm{m}} \uparrow$ & $30-80$ & $\alpha_{\mathrm{b}}^{\prime} \downarrow$ & $130-78$ \\
\hline$\alpha_{\mathrm{b}} \uparrow\left(\beta_{\mathrm{m}} \uparrow\right)$ & $80-114$ & $\alpha_{t}^{\prime} \downarrow$ & $78-74$ \\
\hline$\alpha_{t} \uparrow$ & $114-118$ & $\beta_{\mathrm{m}}^{\prime} \downarrow$ & $74-61$ \\
\hline$\alpha_{\mathrm{b}}^{\prime} \uparrow$ & $118-130$ & & \\
\hline \multicolumn{4}{|c|}{ Phase transition temperature $\left({ }^{\circ} \mathrm{C}\right)$} \\
\hline$T_{\mathrm{c}} \uparrow$ & 116 & $T_{\mathrm{c}} \downarrow$ & 76 \\
\hline
\end{tabular}
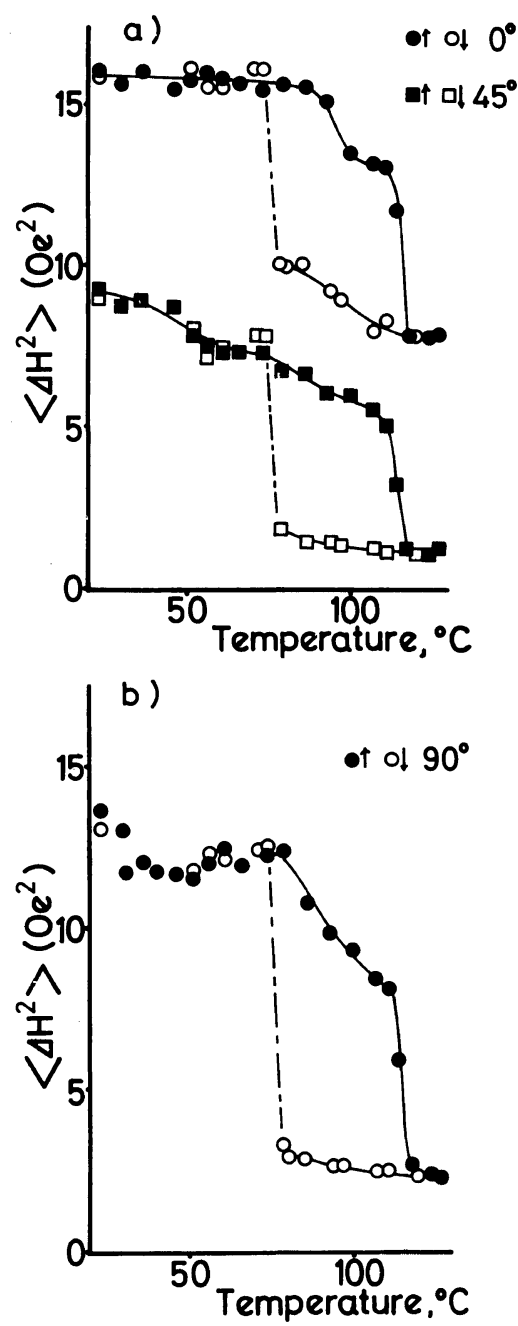

Figure 5. Second moments of $\mathrm{P}(\mathrm{VDF} / \mathrm{TrFE})-72 / 28$ as a function of temperature: (a) $\gamma=0^{\circ}$ and $45^{\circ}$; (b) $\gamma=90^{\circ}$ 


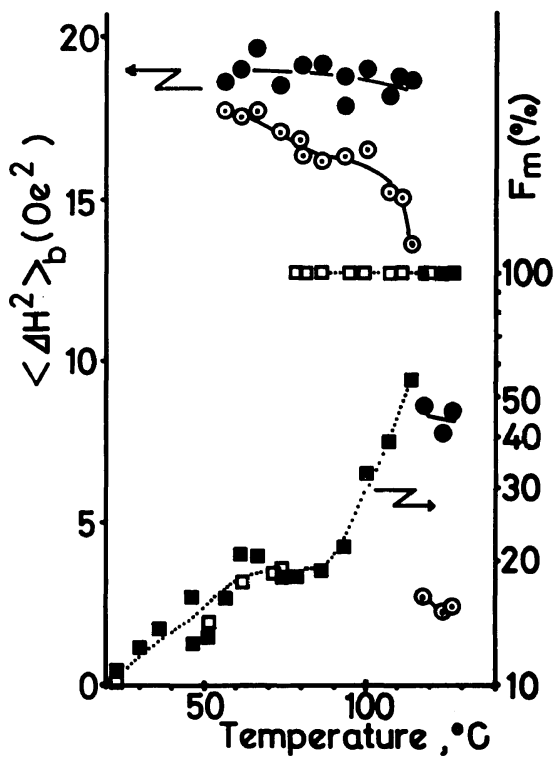

Figure 6. Temperature dependence of the second moment for the broad-line component, $\left\langle\Delta H^{2}\right\rangle_{\mathrm{b}}: \boldsymbol{O}, \gamma=0^{\circ}$; ○, $\gamma=90^{\circ}$, and the mobile fraction, $F_{\mathrm{m}}$ for $\gamma=0^{\circ}: \mathbf{\square}, \uparrow$; $\square, \downarrow$.

temperature. $F_{\mathrm{m}}(\boldsymbol{\square})$ increases by 8 and $30 \%$ in the $\alpha_{\mathrm{m}} \uparrow$ and $\beta_{\mathrm{m}} \uparrow$ (or $\alpha_{\mathrm{b}} \uparrow$ ) regions, respectively. The decrease in $\left\langle\Delta H^{2}\right\rangle_{\mathrm{ob}}\left(90^{\circ}\right)$ in the $\alpha_{\mathrm{b}} \uparrow$ region is as-. sociated with an increase in $F_{\mathrm{m}} . \Delta H_{\mathrm{b}}^{\prime}(\gamma)$ in the $\alpha_{\mathrm{b}}^{\prime} \uparrow$ region is equal to $\Delta H_{\mathrm{m}}(\gamma)$ in the $\beta_{\mathrm{m}} \uparrow$ region near $T_{\mathrm{c}} \uparrow$. Therefore, $F_{\mathrm{m}}$ becomes $100 \%$ above $T_{\mathrm{c}} \uparrow$, indicating that an active chain motion takes place even in the crystalline region. In the $\alpha_{t}^{\prime} \downarrow$ region, $F_{\mathrm{m}}(\square)$ decreases suddenly from 100 to $20 \%$ and return to the original value in the $\uparrow$-process.

\section{DISCUSSION}

Estimations of Line Width and Second Moment for Unoriented $P(V D F / T r F E)$

Table II indicates the line widths, $\overline{\Delta H_{\mathrm{b}}}$ and $\overline{\Delta H_{\mathrm{m}}}$, for unoriented $\mathrm{P}(\mathrm{VDF} / \mathrm{TrFE})$ at room temperature. These are averaged values of $\Delta H_{\mathrm{b}}(\gamma)$ and $\Delta H_{\mathrm{m}}(\gamma)$ for $\gamma=0,45$, and $90^{\circ}$ at $30^{\circ} \mathrm{C}$. The line widths of unoriented PVDF (form I) ${ }^{4}$ and $\mathrm{PTrFE}^{5}$ are included for comparison. There are good agreements between them. From Table II we may consider that the broad and intermediate components are associated with the VDF group and the TrFE group, respectively, in the crystalline phase of $\mathrm{P}(\mathrm{VDF} /$ $\mathrm{TrFE}$ ). This point was ascertained by comparing the
Table II. Line widths of $\mathrm{P}(\mathrm{VDF} / \mathrm{TrFE})-72 / 28$, PVDF from I and PTrFE, at $30^{\circ} \mathrm{C}$

\begin{tabular}{llc}
\hline Sample & \multicolumn{2}{c}{ Line width (Oe) } \\
\hline P(VDF/TrFE) & $13.3^{\mathrm{a}}$ & $8.3^{\mathrm{b}}$ \\
$\begin{array}{c}\text { PVDF (Form-I) } \\
\text { PTrFE }\end{array}$ & 14.0 & 7.8 \\
\hline${ }^{\mathrm{a}}$ Broad width averaged for $\gamma$. \\
b Intermediate width averaged for $\gamma$. \\
c Unoriented sample.
\end{tabular}

second moments of PVDF and PTrFE with that for unoriented $\mathrm{P}(\mathrm{VDF} / \mathrm{TrFE})$ at room temperature. If the Yamagata and Hirota method ${ }^{6}$ is used, the latter can be expressed by

$$
\begin{aligned}
\left\langle\overline{\Delta H^{2}}\right\rangle_{\mathrm{ob}} & =\int_{0}^{2 \pi}\left(S_{0}-S_{2} \sin ^{2} \gamma+S_{4} \sin ^{4} \gamma\right) \sin \gamma \mathrm{d} \gamma \\
& =S_{0}-2 / 3 S_{2}+8 / 15 S_{4}
\end{aligned}
$$

where $S_{0}, S_{2}$, and $S_{4}$ may be determined from observed second moments, $\left\langle\Delta H^{2}\right\rangle_{\mathrm{ob}}$ 's for $\gamma=0,45$, and $90^{\circ}$ at $30^{\circ} \mathrm{C}$.

On the other hand, $\overline{\left\langle\Delta H^{2}\right\rangle}$ for unoriented $\mathrm{P}(\mathrm{VDF} / \mathrm{TrFE})$ can be calculated from

$$
{\overline{\left\langle\Delta H^{2}\right\rangle_{\text {cal }}}}_{=} \chi_{\mathrm{c}}{\overline{\left\langle\Delta H^{2}\right\rangle_{\text {cry }}}}+\left(1-\chi_{\mathrm{c}}\right){\overline{\left\langle\Delta H^{2}\right\rangle_{\text {non }}}}
$$

where ${\overline{\left\langle\Delta H^{2}\right\rangle_{\text {cry }}}}_{\text {and }}{\overline{\left\langle\Delta H^{2}\right\rangle_{\text {non }}}}_{\text {denote the second }}$ moments of the unoriented crystalline and noncrystalline region, respectively, and $\chi_{\mathrm{c}}$, the crystallinity. The crystallinity of $\chi_{c}=0.88$ was determined form the immobile fraction, $1-F_{\mathrm{m}}$, for $\gamma=0^{\circ}$ at $30^{\circ} \mathrm{C} .{\overline{\left\langle\Delta H^{2}\right\rangle_{\text {non }}}}_{\text {is assumed to equal the second }}$ moment for the narrow component, that is, $4.2 \mathrm{Oe}^{2}$ at $30^{\circ} \mathrm{C} .{\overline{\left\langle\Delta H^{2}\right\rangle_{\mathrm{cal}}}}_{\text {is given by }}$

$$
{\overline{\left\langle\Delta H^{2}\right\rangle_{\text {cry }}}}=0.72{\overline{\left\langle\Delta H^{2}\right\rangle}}^{\mathrm{VDF}}+0.28{\overline{\left\langle\Delta H^{2}\right\rangle}}^{\mathrm{TrFE}}
$$

where $\overline{\left\langle\Delta H^{2}\right\rangle}$ VDF and $\overline{\left\langle\Delta H^{2}\right\rangle}{ }^{\mathrm{TrFE}}$ are the second moments calculated for VDF and TrFE monomers, respectively, in which the intra and interchain interactions are taken into account. The details of the calculations are shown in the Appendix. Table III shows good agreement between $\overline{\left\langle\Delta H^{2}\right\rangle_{\mathrm{ob}}}$ given by

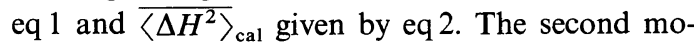
ments of unoriented PVDF (form I) at $-170^{\circ} \mathrm{C}$ and unoriented $\mathrm{PTrFE}$ at $-196^{\circ} \mathrm{C}$ are also shown in Table III. They are in good agreement with $\overline{\left\langle\Delta H^{2}\right\rangle}$ VDF and $\overline{\left\langle\Delta H^{2}\right\rangle^{\text {TrFE}}}$, respectively. These findings support the view that the proton pairs 
Table III. Isotropic second moments of $\mathrm{P}(\mathrm{VDF} / \mathrm{TrFE})-$ $72 / 28$, PVDF from I and PTrFE, at $30^{\circ} \mathrm{C}$

\begin{tabular}{cccc}
\hline & $\begin{array}{c}\left\langle\Delta H^{2}\right\rangle_{\text {cai }} \\
\left(\mathrm{Oe}^{2)}\right.\end{array}$ & $\begin{array}{c}\left\langle\Delta H^{2}\right\rangle_{\text {ob }} \\
\left(\mathrm{Oe}^{2}\right)\end{array}$ \\
\hline$\left\langle\Delta H^{2}\right\rangle^{\mathrm{VDF}}$ & 16.36 & $\mathrm{PVDF}(\text { Form-I })^{\mathrm{a}}$ & 17.1 \\
$\left\langle\Delta H^{2}\right\rangle^{\mathrm{TrFE}}$ & 11.79 & $\mathrm{PTrFE}$ & 12.2 \\
$\mathrm{P}(\mathrm{VDF} / \mathrm{TrFE})$ & 13.7 & $\mathrm{P}(\mathrm{VDF} / \mathrm{TrFE})$ & 12.0 \\
\hline $\mathrm{a}-176^{\circ} \mathrm{C}$. & & & \\
$\mathrm{b}-196^{\circ} \mathrm{C}$. & & & \\
& & &
\end{tabular}

in the monomer group predominantly contribute to $\overline{\left\langle\Delta H^{2}\right\rangle}$ VDF and $\overline{\left\langle\Delta H^{2}\right\rangle}{ }^{\mathrm{TrFE}}$.

\section{Second Moments in the Motional Narrowing Regions and Their $\gamma$-Dependence}

The decrements of second moments, $\delta\left\langle\Delta H^{2}\right\rangle_{\mathrm{ob}}$ in the $\alpha_{\mathrm{m}} \uparrow, \alpha_{\mathrm{b}} \uparrow$, and $\alpha_{\mathrm{t}} \uparrow$ regions, are shown in Figure 7. In Figure $7 \mathrm{a}$ for the $\alpha_{\mathrm{m}} \uparrow$ region, $\delta\left\langle\Delta H^{2}\right\rangle_{\text {ob }}$ has a maximum at $\gamma=45^{\circ}$, which implies a flip-flop motion. The solid line in Figure $7 \mathrm{a}$ shows the $\delta\left\langle\Delta H^{2}\right\rangle_{\text {ob }} v s . \gamma$ curve calculated using a general twosite model, ${ }^{7}$ in which a TrFE group in a $\pm 15^{\circ}$ deflected chain of $\mathrm{P}(\mathrm{VDF} / \mathrm{TrFE})^{2}$ flip-flops between two sites $180^{\circ}$ apart, being accompanied by a rotational oscillation of $40^{\circ}$ in amplitude. The $\gamma$ dependence of the decrement of the second moment for the broad component in the $\alpha_{b} \uparrow$ region, $\delta\left\langle\Delta H^{2}\right\rangle_{\mathrm{b}}$, is shown by the black square ( $\square$ ) in Figure $7 \mathrm{~b}$. This agrees well with the solid line calculated using the oscillation model, in which a
VDF group in the $\pm 15^{\circ}$-deflected chain oscillates about the chain axis with an amplitude of $10^{\circ}$. On the other hand, the $\gamma$-dependences of $\delta\left\langle\Delta H^{2}\right\rangle_{\mathrm{ob}}$ in the $\alpha_{\mathrm{b}} \uparrow, \alpha_{\mathrm{t}} \uparrow$, and $\alpha_{\mathrm{b}}^{\prime} \uparrow$ regions give concave curves. These characteristics cannot be explained by a uniaxial rotation of the rigid chain. The concaveness in these regions may be associated with a motional mode accompanying the conformational change from trans to gauche. The motional broadening of $\Delta H_{\mathrm{m}}$ in the $\beta_{\mathrm{m}} \uparrow$ (or $\alpha_{\mathrm{b}} \uparrow$ ) region (see Figure 3) also suggests that the motion of the TrFE group is accompanied by the conformational change.

\section{$\gamma$-Dependence of the Line Width in the Paraelectric Phase}

Assuming that the $c$-axis is parallel to the $M$-axis as shown in Figure 8 and that the vector $\vec{r}_{i j}$ in a twospin system rotates about the $c$-axis, we can express the line width by the average spin-spin interaction as follows:

$$
\begin{aligned}
\Delta H & =3 / 4 \gamma \hbar / r_{j k}^{3}\left\langle 1-3 \cos ^{2} \theta_{j k}\right\rangle_{\mathrm{av}} \\
& =A\left(1-3 \cos ^{2} \gamma\right)\left(1-3 \cos ^{2} \beta\right)
\end{aligned}
$$

where $\theta_{j k}$ is the angle between $\vec{r}_{j k}$ and $\vec{H}_{0}$, and $\beta$ and $\gamma$ are the angles of $\vec{r}_{j k}$ and $\vec{H}_{0}$ with the $M$-axis, respectively. It is seen from eq 4 that if the vector $\vec{r}_{j k}$ is parallel to the $M$-axis (or the $c$-axis), the relative position of the two nuclei is unaffected by rotation. When $\beta=90^{\circ}$, eq 4 reduces to
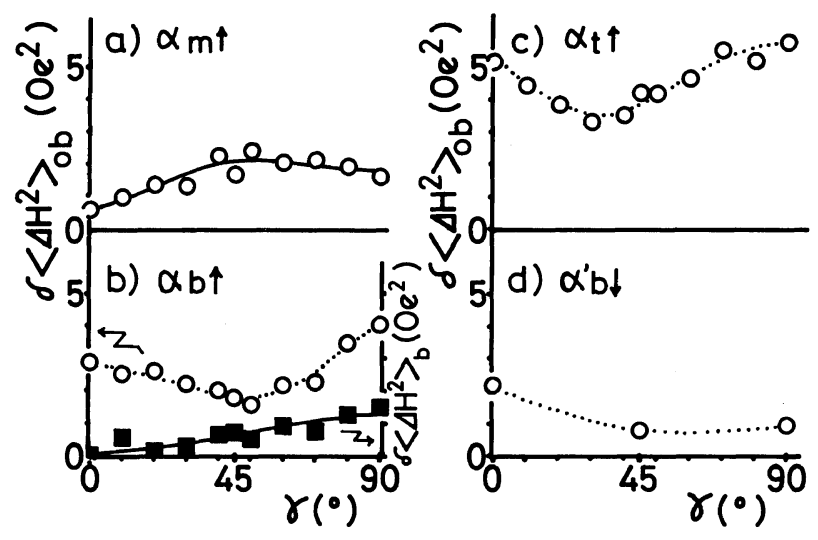

Figure 7. Decrements of the second moment, $\delta\left\langle\Delta H^{2}\right\rangle$, within the following narrowing regions as function of alignment angle $\gamma$; (a) $\alpha_{\mathrm{m}} \uparrow: \bigcirc, \delta\left\langle\Delta H^{2}\right\rangle_{\mathrm{ob}}$ (b) $\alpha_{\mathrm{b}} \uparrow: \bigcirc, \delta\left\langle\Delta H^{2}\right\rangle_{\mathrm{ob}} ; \mathbf{\square}, \delta\left\langle\Delta H^{2}\right\rangle_{\mathrm{b}}$ (c) $\alpha_{\mathrm{t}} \uparrow: \bigcirc$, $\delta\left\langle\Delta H^{2}\right\rangle_{\mathrm{ob}}$ (d) $\alpha_{\mathrm{b}}^{\prime} \downarrow: \bigcirc, \delta\left\langle\Delta H^{2}\right\rangle_{\mathrm{ob}}$. 


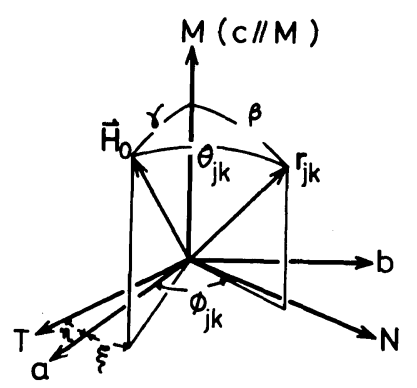

Figure 8. Relation between the crystal coordinates (abc) and the sample coordinates (TNM).

$$
\Delta H=A\left|1-3 \cos ^{2} \gamma\right|
$$

For a two-spin system in which two protons are in the $\left(-\mathrm{CH}_{2}-\mathrm{CF}_{2}-\right)$ unit of the VDF-TrFE copolymer, the line width calculated by eq 5 is shown by the dashed line in Figure 9, and compared with $\Delta H_{\mathrm{b}}^{\prime}$ observed in the $\alpha_{b}^{\prime} \uparrow$ region. The disagreement cannot be explained in terms of a rigid-chain rotation about the chain axis.

Dietz et al. ${ }^{8}$ have pointed out that the exchange narrowing of the ESR absorption line in a onedimensional magnetic substance, $\left(\mathrm{CH}_{3}\right)_{4} \mathrm{NMnCl}_{4}$ (TMMC), can be distinguished definitely from that in three-dimensional magnetic substances. In the former, the relaxation function in the paraelectric phase is given by

$$
\psi(t) \propto \exp \left(\left|1-3 \cos ^{2} \gamma\right|^{4 / 3} t^{3 / 2}-9 \sin \gamma t\right)
$$

The ESR absorption line shape is proportional to the Fourier Transformation of $\psi(t)$, and its line width is expressed by

$$
\Delta H=A\left|1-3 \cos ^{2} \gamma\right|^{4 / 3}
$$

The solid line in Figure 9 is calculated using eq 7 , where the value of $\Delta H$ at $\gamma=0^{\circ}$ is fitted to the experimental value obtained with $\mathrm{P}(\mathrm{VDF} / \mathrm{TrFE})$ at $127^{\circ} \mathrm{C}$. The fitting of the calculated and observed values is satisfactory. This may be interpreted by an analogy in spin-Brownian motion between TMMC and $\mathrm{P}(\mathrm{VDF} / \mathrm{TrFE})$ as follows: The chain motion in $\mathrm{P}(\mathrm{VDF} / \mathrm{TrFE})$ is one-dimensional in the paraelectric phase, since above $T_{\mathfrak{c}}$, the interchain interactions are extremely weak in comparison with the intrachain interactions. Hence, the energies localized about conformational defects diffuse mostly along the chain, and the local magnetic field fluctuates in a similar way to that of TMMC in-

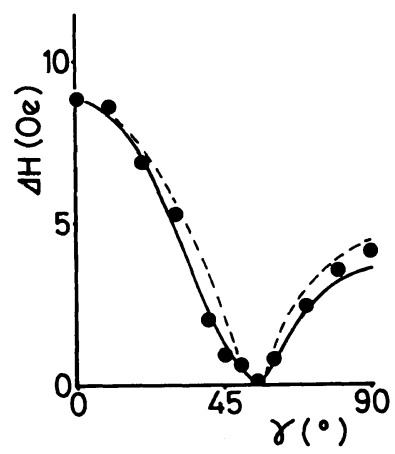

Figure 9. Angular dependence of the broad width in the derivative curve of $\mathrm{P}(\mathrm{VDF} / \mathrm{TrFE})-72 / 28$, at $127^{\circ} \mathrm{C}$ in the $\quad \alpha_{b}^{\prime} \uparrow$ region:,$- \quad 9\left|3 \cos ^{2} \gamma-1\right|^{4 / 3}$; $\quad$-..---, $9\left|3 \cos ^{2} \gamma-1\right|$.

vestigated by Dietz et al. ${ }^{8}$ Such localized diffusion of conformational defects in the chain may also take place in the $\alpha_{b} \uparrow, \alpha_{t} \uparrow$, and $\alpha_{b}^{\prime} \downarrow$ regions, and strongly depend on the motional mode accompanying the comformational change from trans to gauche, as suggested by the concave curve in the $\delta\left\langle\Delta H^{2}\right\rangle_{\mathrm{ob}} v s$. $\gamma$ relation.

\section{CONCLUSION}

Multipeaks appeared in the NMR differential aborption line of a VDF-TrFE copolymer (72:28), and some of them indicated a $\gamma$-dependence. In the ferroelectric phase, the broad and intermediate peaks may be attributed to the VDF and TrFE groups, respectively. Various narrowing regions appeared in the temperature range from 23 to $130^{\circ} \mathrm{C}$. The decrement in the second moment taking place in these regions showed a strong $\gamma$-dependence, suggesting the occurrence of chain motion in the crystalline region. The mechanisms of the chain motion in these regions are classified as follows:

(1) $\alpha_{m} \uparrow$ Process: a flip-flop motion of a TrFE group between two sites $180^{\circ}$ apart, accompanying a rotational oscillation of $40^{\circ}$ in amplitude.

(2) $\alpha_{b} \uparrow, \alpha_{t} \uparrow$ and $\alpha_{b}^{\prime} \downarrow$ Processes: motional modes different from the above-mentioned two kinds of chain motion, but associated with the conformational change from trans to gauche bond.

In particular, it should be noted that in the paraelectic phase, the fluctuation of local magnetic field shows a one-dimensional characteristic, since energies localized about conformational defects dif- 
fuse mostly along the chain.

\section{APPENDIX}

\section{Calculation of the Second Moment of Unoriented $P(V D F / \operatorname{Tr} F E)$}

We consider the contribution of the VDF and TrFE monomer groups in an unoriented random copolymer P(VDF/TrFE) to the NMR second moment. The nuclear dipole-dipole interaction in the region, where the distance between $i$ and $j$ nuclei is larger than $5 \AA$, hardly contributes to the second moment. Therefore, a pentad monomer sequence chain is taken into account for the intrachain second moment $\left\langle\Delta H^{2}\right\rangle_{\text {intra }}$, and for the interchain second moment $\left\langle\Delta H^{2}\right\rangle_{\text {inter }}$, the nearest neighbor chains are considered as follows:

(i) Pentad Monomer Sequence Distribution in the Intrachain. We consider a pentad monomer sequence chain in which the VDF monomer (or the TrFE one) is at its center. Since $\mathrm{P}(\mathrm{VDF} / \mathrm{TrFE})$ is a random copolymer, ${ }^{9}$ the probability that any one monomer in the pentad sequence chain is VDF (or TrFE) is given by $p$ (or $q$ ), where $p$ (or $q$ ) is the mole percent of VDF (or TrFE). In the pentad sequence chain, the probability, $P^{(i)}(n)$, that $n$ of $N$ monomers $(N=4)$ except the center monomer is VDF is given by

$$
P^{(i)}(n)=\left(\begin{array}{l}
N \\
n
\end{array}\right) p^{n} q^{N-n} \quad(i=\mathrm{VDF} \text { or } \operatorname{TrFE})
$$

(ii) Distribution of Monomers in the Nearest Neighbor Chains. We assume that there is no short range order in the nearest neighbor chains, and consider that the $M$ nearest neighbor chains take either a VDF or TrFE monomer at their centers. The probability $Q^{(j)}(m)$ that $m$ of the $M$ monomers at the centers in the nearest neighbor chains are VDF monomers is given by

$$
Q^{(j)}(m)=\left(\begin{array}{c}
M \\
m
\end{array}\right) p^{m} q^{M-m} \quad(j=\mathrm{VDF} \text { or } \operatorname{TrFE})(\mathrm{A}-2)
$$

For the present case, $N=4, M=6, p=0.72$, and $q=$ 0.28 .

(iii) Second Moment of $P(V D F / T r F E)$ Crystal. $\left\langle\Delta H^{2}\right\rangle_{\text {cry }}$ is given by eq 3 in terms of $\left\langle\Delta H^{2}\right\rangle^{\text {VDF }}$ and $\left\langle\Delta H^{2}\right\rangle^{\mathrm{TrFE}}$ which are in turn given by

$$
\begin{gathered}
\left\langle\Delta \mathrm{H}^{2}\right\rangle^{\mathrm{VDF}}=\sum_{n}^{4} P^{(i)}(n)\left\langle\Delta H^{2}\right\rangle_{\mathrm{intra}}^{\mathrm{VDF}} \\
+\sum_{m}^{6} Q^{(i)}(m)\left\langle\Delta H^{2}\right\rangle_{\mathrm{inter}}^{\mathrm{VDF}} \\
(i=\mathrm{VDF}) \\
\left\langle\Delta H^{2}\right\rangle^{\mathrm{TrFE}}=\sum_{n}^{4} P^{(j)}(n)\left\langle\Delta H^{2}\right\rangle_{\mathrm{intra}}^{\mathrm{TrFE}} \\
+\sum_{n}^{6} Q^{(j)}(m)\left\langle\Delta H^{2}\right\rangle_{\text {inter }}^{\mathrm{TrFE}} \\
(j=\mathrm{TrFE})
\end{gathered}
$$

The calculations of $\left\langle\Delta H^{2}\right\rangle^{\mathrm{VDF}}$ and $\left\langle\Delta H^{2}\right\rangle^{\mathrm{TrFE}}$ were made in the $\pm 15^{\circ}$-deflected chain, which was ascertained by Tashiro et al. ${ }^{2}$

\section{REFERENCES}

1. Andrew J. Lovinger, G. T. Davis, T. Furukawa, and M. G. Broadhurst, Macromolecules, 15, 323 (1982); G. T. Davis, T. Furukawa, Andrew J. Lovinger, and M. G. Broadhurst, Macromolecules, 15, 329 (1982).

2. K. Tashiro, K. Takano, M. Kobayashi, Y. Chatani, and H. Tadokoro, Polymer, 22, 1312 (1981).

3. T. Furukawa, M. Date, E. Fukada, Y. Tjitsu, and A. Chiba, Jpn. J. Appl. Phys., 19, L109 (1980).

4. F. Ishii, T. Sawatari, and A. Odajima, Jpn. J. Appl. Phys., 21, L251 (1982).

5. W. P. Slichter, J. Polym. Sci., 24, 173 (1957).

6. K. Yamagata and S. Hirota, Oyo Butsuri, 30, 261 (1961).

7. H. G. Olf and A. Peterlin, J. Polym. Sci., A-2, 8, 753 (1970).

8. R. E. Dietz, F. R. Merritt, R. Dingle, D. Hone, B. G. Silibernagel, and P. M. Richards, Phys. Rev. Lett., 9, 1186 (1971)

9. T. Yagi and M. Tatemoto, Polym. J., 11, 429 (1979). 